\title{
Epithelial-mesenchymal transition of A549 cells is enhanced by co-cultured with THP-1 macrophages under hypoxic conditions
}

\author{
Akane Sueki $^{\mathrm{a}, \mathrm{b}}$, Kazuyuki Matsuda ${ }^{\mathrm{a}, *}$, Chinami Iwashita ${ }^{\mathrm{c}}$, Chiaki Taira ${ }^{\mathrm{a}, \mathrm{b}}$, Nau Ishimine ${ }^{\mathrm{a}, \mathrm{b}}$, \\ Shohei Shigeto ${ }^{\mathrm{a}, \mathrm{b}}$, Kenji Kawasaki ${ }^{\mathrm{a}}$, Mitsutoshi Sugano ${ }^{\mathrm{a}}$, Hiroshi Yamamoto ${ }^{\mathrm{d}}$, Takayuki Honda ${ }^{\mathrm{a}, \mathrm{b}}$ \\ ${ }^{a}$ Department of Laboratory Medicine, Shinshu University Hospital, Matsumoto, Japan \\ ${ }^{\mathrm{b}}$ Department of Laboratory Medicine, Shinshu University School of Medicine, Matsumoto, Japan \\ ${ }^{\mathrm{c}}$ Department of Health and Medical Sciences, Graduate School of Medicine, Shinshu University, Matsumoto, Japan \\ ${ }^{\mathrm{d}}$ Department of Internal Medicine, Shinshu University School of Medicine, Matsumoto, Japan
}

\section{A R T I C L E I N F O}

\section{Article history:}

Received 6 October 2014

Available online 14 October 2014

\section{Keywords:}

Hypoxia

Epithelial-mesenchymal transition

Type II pneumocytes

Macrophages

Idiopathic pulmonary fibrosis

\begin{abstract}
A B S T R A C T
Epithelial-mesenchymal transition (EMT) is associated with pulmonary fibrosis, including idiopathic pulmonary fibrosis (IPF). In this study, we investigated EMT of human pulmonary epithelial-derived cells (A549). A549 cells was either cultured by itself or co-cultured with THP-1 macrophages under normoxic $\left(21 \% \mathrm{O}_{2}\right)$ and hypoxic $\left(2 \% \mathrm{O}_{2}\right)$ conditions. We evaluated the presence of EMT by determining the expression of EMT markers, E-cadherin, vimentin, and fibronectin. To determine the role of TGF- $\beta 1$ and IL- $1 \beta$ in EMT of the A549 cells, we analyzed the effects of blocking their activity with TGF- $\beta 1$ inhibitor or IL- $1 \beta$ neutralizing antibody respectively. The A549 cells presented EMT when they were co-cultured with THP-1 macrophages. The EMT of the A549 cells co-cultured with THP-1 macrophages was exacerbated under hypoxia. In addition, the EMT were prevented by the addition of TGF- $\beta 1$ type I receptor kinase inhibitor. The hypoxic condition increased the mRNA levels of TGF- $\beta 1$ in A549 cells and THP- 1 macrophages and that of IL-1 $\beta$ in THP-1 macrophages when each cells were co-cultured. Anti-IL-1 $\beta$ neutralizing antibody attenuated TGF- $\beta 1$ secretion in co-culture media under hypoxic conditions. Thus, the IL- $1 \beta$ from THP- 1 macrophages up-regulated the TGF- $\beta 1$ from A549 cells and THP- 1 macrophages, and then the TGF- $\beta 1$ from both cells induced and promoted the EMT of A549 cells when they were co-cultured under hypoxia. Together, these results demonstrate that the interaction between type II pneumocytes and macrophages under hypoxia is necessary for the development of pulmonary fibrosis.
\end{abstract}

(C) 2014 Elsevier Inc. All rights reserved.

\section{Introduction}

Idiopathic pulmonary fibrosis (IPF), the most common form of idiopathic interstitial pneumonia (IIP) is a progressive disorder with unknown etiology. Patchy areas of fibrosis associated with exaggerated accumulation of extracellular matrix (ECM) mark the IPF pathology $[1,2]$. The IPF possibly develops because of recurring injury to the alveolar epithelium with concomitant dysregulation of cellular homeostasis, followed by erroneous repair of the epithelial damage. However, the exact mechanisms underlying the IPF are unknown.

Recent reports suggest that the alveolar epithelial cells undergo epithelial-mesenchymal transition (EMT), and in turn serve as a

* Corresponding author at: Department of Laboratory Medicine, Shinshu University Hospital, Asahi 3-1-1, Matsumoto 390-8621, Japan. Fax: +81 263345316.

E-mail address: kmatsuda@shinshu-u.ac.jp (K. Matsuda). source of fibroblasts that are associated with the lung fibrogenesis [3]. EMT is characterized by an increase in mesenchymal markers (e.g., vimentin and fibronectin) and loss of epithelial markers (e.g., E-cadherin) [4]. In fact, the loss of alveolar epithelial and gain of mesenchymal markers have been observed in the alveolar epithelium of lung fibrotic animal models and IPF patients $[5,6]$. Because of the role of TGF- $\beta 1$ in EMT, it is not surprising that it plays an important role in the development of alveolar EMT associated with pathological fibrosis [3]. The overexpression of TGF- $\beta 1$ in rats induces severe pulmonary fibrosis and persistence of the disease [7]. TGF- $\beta 1$ alters expression of proteins that regulate the integrity epithelial tissues, leading to cytoskeletal rearrangement and morphological changes. In fact, alveolar epithelial cells that lay over the fibroblastic foci often show decreased or loss of E-cadherin expression. In addition, the immune macrophage cells significantly increase in IPF as seen with the cell fractionation of the bronchoalveolar lavage (BAL) fluids from IPF patients [8-10]. 
Moreover, the proinflammatory cytokines derived from mouse macrophages increase the expression of TGF- $\beta$ and induce EMT of A549, human type II alveolar epithelial cell line [11].

Fibroblast proliferation, inflammatory cell infiltration, and interstitial thickening can potentially combine with alveolar ventilation defects, leading to increased oxygen consumption, and restriction of the oxygen supply to the injured lung, thus, resulting in localized tissue hypoxia [12]. Hypoxia induced macrophage activation alters gene expression through cooperative interaction between nuclear factor kappa-light-chain-enhancer of activated $\mathrm{B}$ cells (NF- $\kappa \mathrm{B}$ ) and hypoxia inducible factors (HIFs) [13]. Ultimately, alveolar hypoxia causes structural changes in the lung, which leads to chronic fibroproliferation, resulting in inflammation and thickening of the alveolar wall [14].

In this study, we examined the induction of EMT in type II pneumocytes (A549 cells) when co-cultured with THP-1 macrophages under normoxic and hypoxic conditions.

\section{Materials and methods}

\subsection{Cell culture}

A549 and THP-1 cell lines were purchased from the Japanese Cancer Research Bank (JCRB, Tokyo, Japan) and the American Type Culture Collection (ATCC, Rockville, MD) respectively. A549 and THP-1 cells were maintained in $10 \%$ fetal bovine serum (FBS) supplemented Dulbecco's modified Eagle's medium (DMEM, Nacalai Tesque, Kyoto, Japan) and RPMI1640 (Nacalai Tesque, Kyoto, Japan) respectively. Macrophage differentiation of the THP-1 cells was triggered by adding $500 \mathrm{ng} / \mathrm{ml}$ phorbol 12-myristate 13-acetate (PMA, Sigma, St. Louis, MO) for 4 days.

A549 cells and THP-1 macrophages were co-cultured using a Millicell Hanging Cell Culture Insert (Millipore, Billerica, MA) with a membrane pore size of $1.0 \mu \mathrm{m}$ (Fig. 1A). Hypoxic conditions were achieved by adjusting the incubator (ASTEC, Fukuoka, Japan) atmosphere to $5 \% \mathrm{CO}_{2}, 2 \% \mathrm{O}_{2}$, and $93 \% \mathrm{~N}_{2}$, and $95 \%$ air and $5 \%$ $\mathrm{CO}_{2}$ (Incubator - MCO-175; SANYO, Tokyo, Japan) were the conditions for normoxia.

\subsection{TGF- $\beta 1$ receptor inhibition and IL-1 $\beta$ neutralization}

For the inhibition assays, the A549 cells and the THP-1 macrophages were co-cultured in the presence of either in $10 \mu \mathrm{mol} / \mathrm{L}$ TGF- $\beta 1$ type I receptor inhibitor, SB-431542 (Sigma, St. Louis, $\mathrm{MO}$ ) or $385 \mu \mathrm{mol} / \mathrm{L}$ DMSO, which served as a control under hypoxic conditions at $37^{\circ} \mathrm{C}$ for 7 days to evaluate E-cadherin, vimentin and fibronectin expression.

For neutralization assays, the A549 cells and THP-1 macrophages were grown in the presence of either $10 \mu \mathrm{g} / \mathrm{ml}$ anti-mouse IL-1 $\beta$ neutralizing antibody or mouse control IgG (R \& D Systems, UK) under hypoxic conditions at $37^{\circ} \mathrm{C}$ for 2 days to evaluate the amount of total TGF- $\beta 1$ secretion.

\subsection{Real-time quantitative polymerase chain reaction (PCR)}

Total RNA was extracted from A549 cells and THP-1 macrophages using the QIAamp ${ }^{\circledR}$ RNA Blood Mini Kit (Qiagen, Valencia, CA). Quantitative real-time RT-PCR was performed with QuantStudio $12 \mathrm{~K}$ flex systems (Life Technologies, Carlsbad, CA) using Life Technologies TaqMan $^{\circledR}$ Gene Expression Assays for TGF $\beta 1$ (Hs00998133_m1), IL-1 $\beta$ (Hs01555410_m1), and $\beta$-actin (Hs99999903_m1). The samples were assessed by $2^{-\Delta \Delta C t}$ relative quantitative analysis to determine the differences in the expression levels. All experiments were analyzed in triplicate or quadruplicate samples.

\subsection{Immunoblotting}

Cultured A549 cells were harvested and sonicated in 200$500 \mu \mathrm{l}$ PBS, followed by estimation of total protein concentrations using the Pierce BCA Protein Assay Kit (Thermo Scientific). The protein lysates were denatured in sample buffer containing 5\% 2mercaptoethanol, subjected to electrophoresis on $8-16 \%$ SDSPAGE mini gel (Tefco, Japan), and transferred to a nitrocellulose membrane. The membrane was blocked in $5 \%$ non-fat dry milk dissolved in $0.1 \%$ Tween-20-PBS prior to incubation with primary antibodies. The primary antibodies used in this study were as follows: mouse anti-E-cadherin (1:500, BD Biosciences, San Jose, CA), mouse anti-vimentin (1:100, Dako, Copenhagen, Denmark), rabbit anti-fibronectin and mouse anti- $\beta$-actin (1:2000, Sigma). Following incubation with HRP conjugated secondary antibodies, the proteins were detected by ECL-PLUS (GE Healthcare, Amersham, UK), and the band density was measured using Image software and normalized to $\beta$-actin. The immunoblotting data are representative of three or four independent experiments.

\subsection{Immunofluorescence staining}

A549 cells were fixed with 4\% paraformaldehyde in PBS, permeabilized with $0.1 \%$ Triton-X, blocked with $3 \%$ BSA in PBS. The cells were then incubated with primary antibodies against E-cadherin (1:100, BD Biosciences), vimentin (1:100, Dako) or fibronectin (1:800, Sigma), followed by incubation with FITC or Alexa 555conjugated secondary antibodies. After mounting the cells in

A

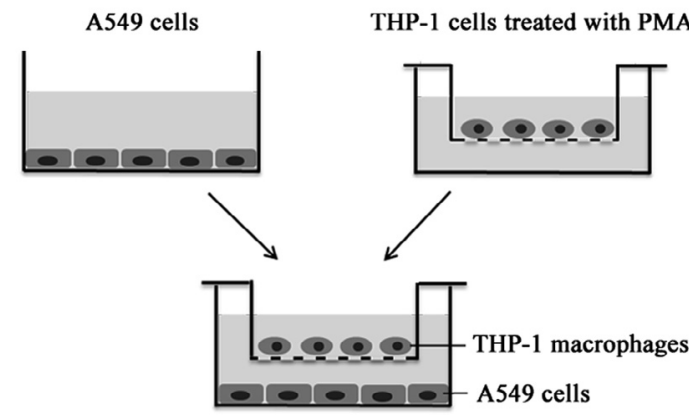

B

Normoxia

Hypoxia

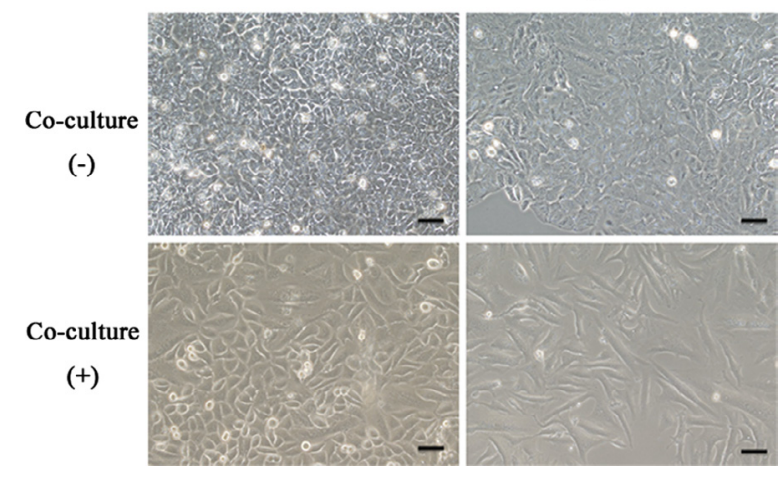

Fig. 1. Hypoxia promoted morphological changes of A549 cells co-cultured with THP-1 macrophages. (A) Schematic representation of the indirect co-culture system of A549 cells and THP-1 macrophages. A549 cells were cultured alone at the bottom of 6-well plate for a day. THP-1 cells were plated in the apical compartment of a 6well Millicell Hanging Cell Culture Insert with a membrane pore size of $1.0 \mu \mathrm{m}$ and were treated with $500 \mathrm{ng} / \mathrm{mL}$ PMA for 4 days to differentiate into macrophage-like forms. Then, the cell culture inserts containing THP- 1 macrophages were transferred to the plates containing A549 cells. (B) A549 cells were co-cultured with THP-1 macrophages under normoxic or hypoxic conditions for 7 days. Phase contrast images of A549 cells under the above conditions. Scale bar $-50 \mu \mathrm{m}$. 
A
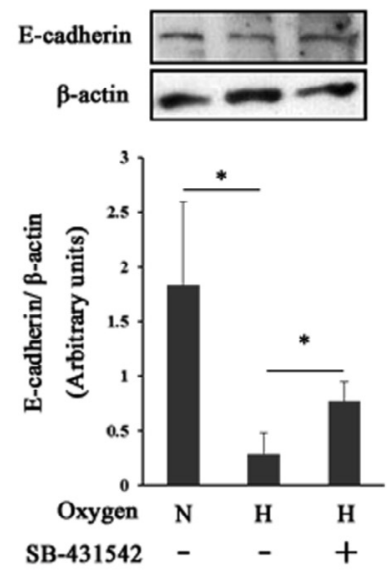

B
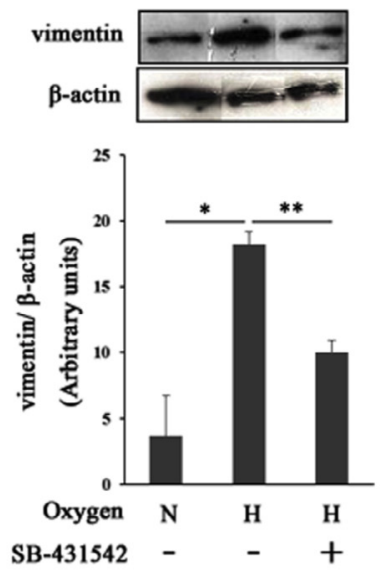

C
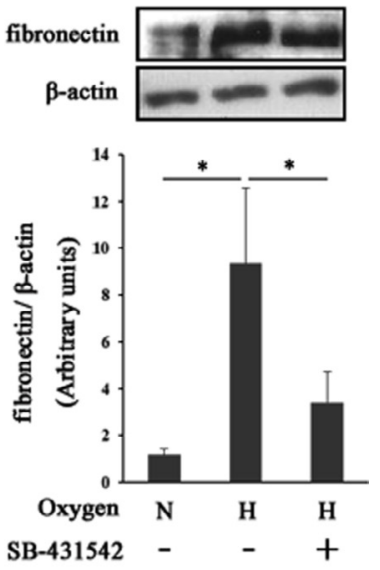

D

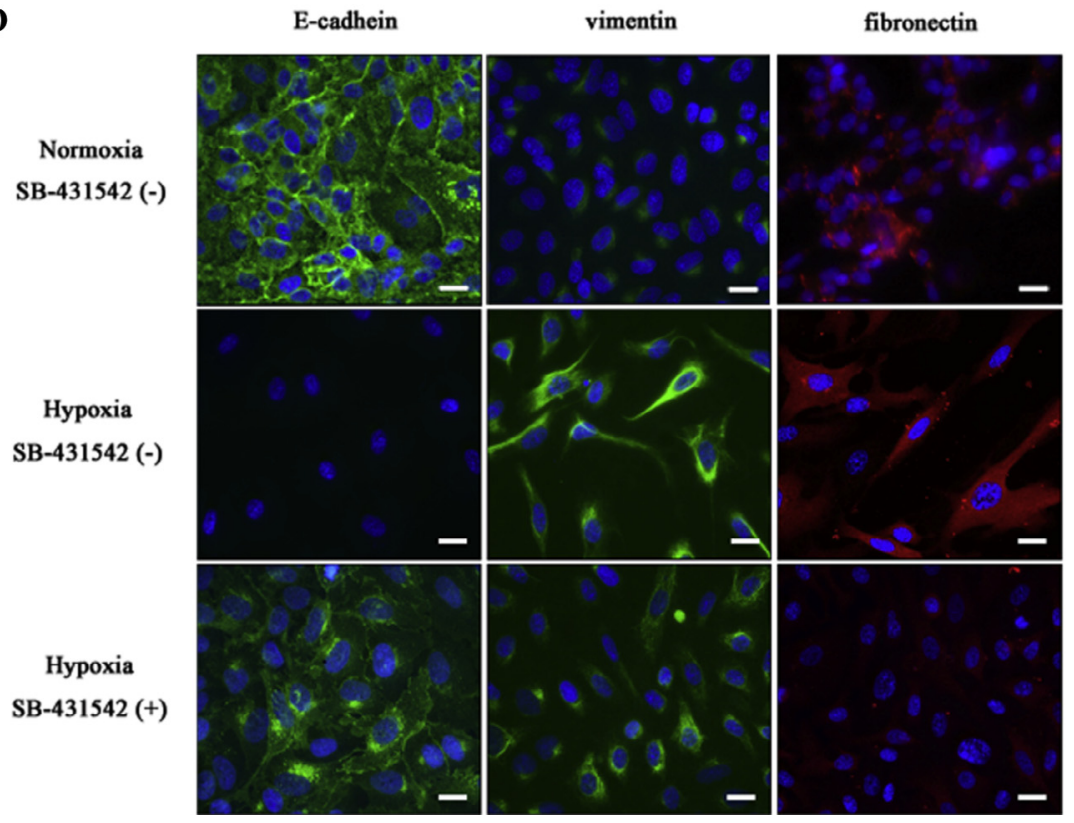

Fig. 2. EMT of A549 cells exacerbated by hypoxia was TGF- $\beta 1$ dependent. A549 cells co-cultured with the THP- 1 macrophages under normoxic or hypoxic conditions were treated with $10 \mu \mathrm{M}$ SB431542 or $385 \mu \mathrm{M}$ DMSO control for 7 days. (A-C) Immunoblots were obtained using anti-E-cadherin, anti-vimentin, anti-fibronectin, and anti- $\beta$-actin antibodies (upper), and the relative amounts quantified by the band density (lower) are shown. $\beta$-actin was used as loading control. Data are mean \pm SD ( $n \geqslant 3$ ). $N-$ normoxia; $\mathrm{H}$ - hypoxia. ${ }^{*} \mathrm{P}<0.05 ;{ }^{* *} P<0.01$. (D) Immunofluorescence staining of vimentin, fibronectin and E-cadherin in A549 cells. Nuclei were counterstained with DAPI (blue). Scale bar $-20 \mu \mathrm{m}$.

SlowFade Gold anti-fade reagent with DAPI (Invitrogen, Carlsbad, CA), images were captured using Axioplan 2 imaging microscope (Zeiss, Oberkochen, Germany).

\subsection{Measurement of total TGF- $\beta 1$ secretion}

Secretion of total TGF- $\beta 1$ in the media was measured by ELISA kit (R \& D Systems, UK). Each sample was assayed in triplicate according to the manufacturer's instructions. The amount of total TGF- $\beta 1$ was quantified by measuring its absorption at $450 \mathrm{~nm}$ with a Spectra Max Plus microplate spectrophotometer (Molecular Devices, Sunnyvale, CA).

\subsection{Statistical analysis}

Statistical analysis was performed using PASW Statistics 21 software. Data were expressed as mean \pm SD. Kruskal-Wallis statistics was used for multiple data comparisons, and independent t-test was performed between the two groups. A p-value $<0.05$ was considered statistically significant.

\section{Results}

3.1. Hypoxic conditions promoted morphological changes of A549 cells co-cultured with THP-1 macrophages

To determine if A549 cells underwent EMT under hypoxic conditions, and if the presence of THP- 1 macrophages affects EMT of A549 cells, we investigated the morphological changes of A549 cells when single cultured or co-cultured with THP-1 macrophages under normoxia or hypoxia for 7 days (Fig. 1). A549 cells morphology is slightly altered to be elongated and scattered when cocultured with THP-1 macrophages (Fig. 1B bottom columns). The morphological changes of A549 cells co-cultured with THP-1 
A

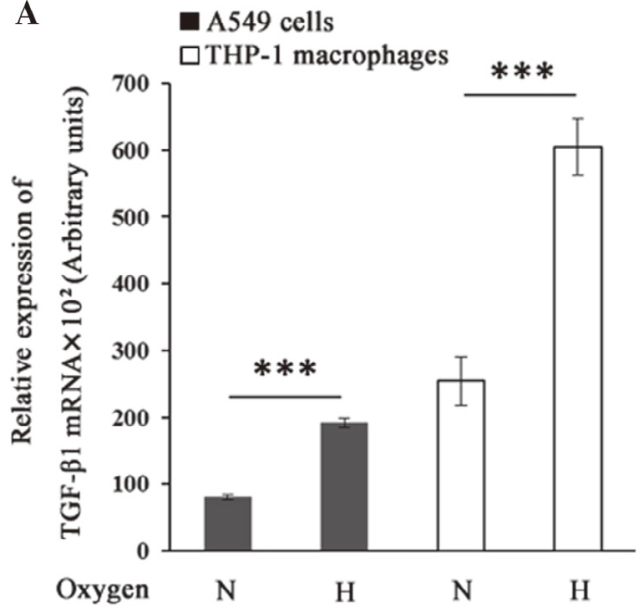

$\mathbf{B}$

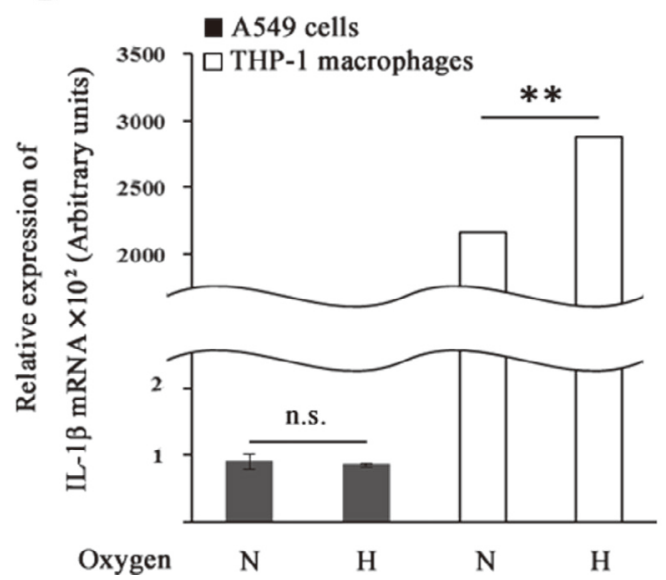

C

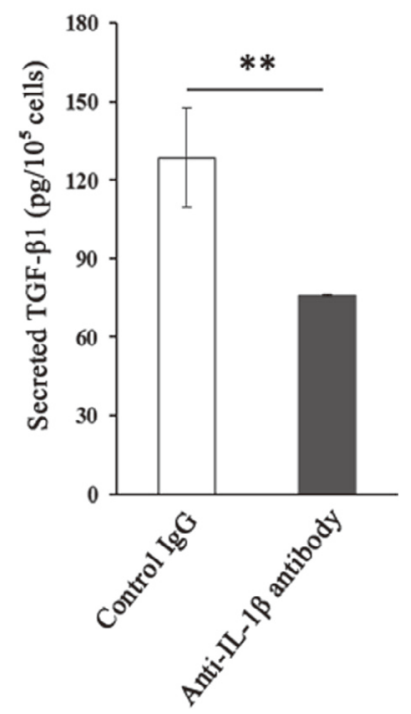

Fig. 3. Interaction of IL- $1 \beta$ and TGF- $\beta 1$ induced EMT of A549 cells co-cultured with THP- 1 macrophages under hypoxic conditions. (A) A549 cells were co-cultured with THP-1 macrophages under normoxic or hypoxic conditions for 2 days. The amount of TGF- $\beta 1$ mRNA in A549 cells and THP- 1 macrophages were determined with quantitative realtime PCR. Data are mean $\pm \mathrm{SD}(n=3)$. $\mathrm{N}$ - normoxia; $\mathrm{H}$ - hypoxia. ${ }^{* * *} \mathrm{P}<0.001$. (B) THP- 1 macrophage cells were co-cultured with A549 under normoxic or hypoxic conditions for $3 \mathrm{~h}$. Expression of IL-1 $\beta$ mRNA in A549 cells and THP-1 macrophages were analyzed by real-time PCR. Data are mean \pm SD $(n=4)$. N - normoxia; $\mathrm{H}-\mathrm{hypoxia}$. ${ }^{* *} P<0.01$. n.s., not significant. (C) A549 cells co-cultured with the THP-1 macrophages under hypoxic conditions were treated with $10 \mu \mathrm{g} / \mathrm{ml}$ anti-mouse IL-1 $\beta$ neutralizing antibody or mouse control IgG for 2 days. The amount of total TGF- $\beta 1$ secretion in co-cultured media was measured by ELISA. Data are mean \pm SD $(n=3)$. ${ }^{* *} P<0.01$ vs. control.

macrophages were more remarkable under hypoxia than normoxia (Fig. 1B right bottom column).

\subsection{EMT of A549 cells exacerbated by hypoxia was TGF- $\beta 1$ dependent}

To study the effects of $\mathrm{O}_{2}$ concentration on the EMT of A549 cells co-cultured with THP-1 macrophages at a molecular level, we examined the expression levels of epithelial marker, E-cadherin and mesenchymal markers, vimentin and fibronectin in A549 cells by immunoblotting and immunofluorescence staining. Upon coculture with THP-1 macrophages under hypoxic conditions for 7 days, significant decrease of E-cadherin and increase of vimentin and fibronectin in A549 cells were observed compared to that under normoxic conditions by immunoblotting and immunofluorescence staining (Fig. 2). Next, we employed the approaches to inhibit TGF- $\beta 1$ type I receptor activity to investigate the role of TGF- $\beta 1$ in our model. Treatment of the cells with the inhibitor of TGF- $\beta 1$ type I receptor activity partially reverses the hypoxiainduced reduction of E-cadherin expression in A549 cells (Fig. 2A). Similarly, hypoxia-induced gain in vimentin and fibronectin expression is prevented by the SB-431542 (TGF- $\beta 1$ inhibitor) (Fig. 2B and C). Furthermore, immunofluorescence staining confirmed the effects of the inhibitor of TGF- $\beta 1$ type I receptor activity (Fig. 2D).

\subsection{Interaction of IL- $1 \beta$ and TGF- $\beta 1$ induced EMT of A549 cells co-cultured with THP-1 macrophages under hypoxic conditions}

To reveal the effect of hypoxic conditions on the expression of TGF- $\beta 1$ in A549 cells and THP- 1 macrophages, we examined the expression of TGF- $\beta 1$ mRNA in each cells. In the presence of co-culture for 2 days, hypoxic conditions significantly elevated the mRNA levels of TGF- $\beta 1$ in A549 cells and THP- 1 macrophages (Fig. 3A). We also examined the effect of the hypoxic conditions on the expression of IL- $1 \beta$ mRNA, upstream regulator of TGF- $\beta 1$. A549 cells expressed a trace amount of IL-1 $\beta$, on the other hand, THP-1 macrophages expressed relatively large amounts of IL- $1 \beta$ compared to A549 cells. The expression of IL- $1 \beta$ mRNA significantly increased in THP-1 macrophages, but not in A549 cells, when they were co-cultured under hypoxic conditions for $3 \mathrm{~h}$ (Fig. 3B). After determining the expression of IL-1 $\beta$ mRNA under all the above conditions, we sought to ask if blocking IL-1 $\beta$ would affect TGF- $\beta 1$ expression. In fact, treatment with anti-IL-1 $\beta$ neutralizing antibody for 2 days decreased total TGF- $\beta 1$ secretion into the co-culture medium under hypoxic conditions (Fig. 3C). 


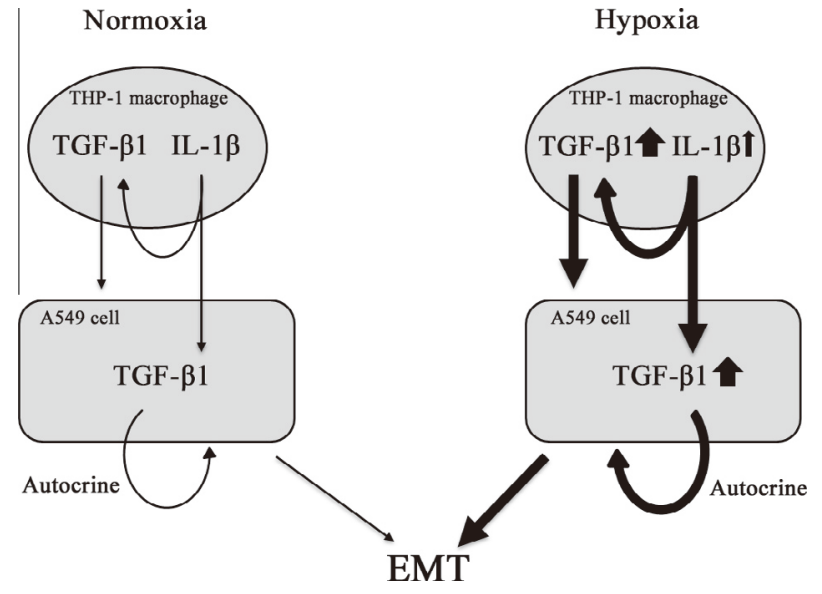

Fig. 4. Model for mechanisms underlying EMT of A549 cells. Hypoxia may act on the THP-1 macrophages co-cultured with A549 cells to increase the expression of IL-1 $\beta$. The more increased IL- $1 \beta$ secretion from THP- 1 macrophages under hypoxia may stimulate THP- 1 macrophages and A549 cells to produce TGF- $\beta 1$ via autocrine or paracrine manner. Therefore, when co-cultured with macrophages under hypoxic condition, but not normoxic condition, significant increase of TGF- $\beta 1$ in A549 cells and THP-1 macrophages may result in induction and promotion of the EMT of A549 cells.

\section{Discussion}

In this study, we evaluated the EMT of A549 cells when single cultured or co-cultured with THP-1 macrophages under normoxia or hypoxia by analyzing morphological changes and the expression of epithelial (E-cadherin) and mesenchymal (vimentin and fibronectin) markers. A549 cells co-cultured with THP-1 macrophages presented the morphological changes that imply a mesenchymal phenotype as epithelial cells lose their cell-to-cell junctions and cell polarity, and acquire a mesenchymal shape during EMT [15] (Fig. 1B bottom columns). The morphological changes of A549 cells co-cultured with THP-1 macrophages became more apparent under hypoxia than normoxia (Fig. 1B right bottom column). We observed more significant downregulation of E-cadherin and an upregulation of vimentin and fibronectin in A549 cells, which were consistent with EMT signatures [4], when they were co-cultured with THP-1 macrophages under hypoxia compared to that under normoxia (Fig. 2). These results indicated that the interaction between A549 cells and THP-1 macrophages under normoxia has a potential to induce EMT of A549 cells, and the interaction under hypoxia exacerbated the EMT of A549 cells.

TGF- $\beta 1$ plays an important role in the initiation and maintenance of EMT of lung epithelial cells in both animal models and humans [3]. In this study, inhibiting TGF- $\beta 1$ type I receptor kinase prevented A549 cells from undergoing EMT (Fig. 2). More importantly, the co-culture under hypoxic conditions significantly increased the expression of TGF- $\beta 1$ mRNA in A549 cells and THP-1 macrophages (Fig. 3A). Alveolar hypoxia in human lung diseases culminates in acute lung injury and pulmonary fibrosis [16,17]. It induces macrophage activation, production of inflammatory and fibrotic cytokines [18], and acts on pneumocytes to disrupt cytoskeleton integrity [19-21]. In rats, macrophage number in the BAL increases under hypoxia-induced inflammation [18], indicating that inflammation caused by hypoxia may directly contribute to pulmonary fibrosis pathogenesis. In pulmonary fibrosis, epithelial stress may induce alveolar epithelial cells to secrete TGF- $\beta$. Abnormal activation of TGF- $\beta$ leads to trans-differentiation of quiescent fibroblasts into myofibroblasts, and excessive production of fibrillar collagens $[1,22,23]$ as observed in IPF patients [24]. Additionally, increased elevation of TGF- $\beta 1$ under hypoxic conditions can lead to rapid and pronounced EMT [25].
Co-treatment of A549 cells with TGF- $\beta 1$ and IL-1 $\beta$ increases EMT [26]. This study demonstrates that IL-1 $\beta$ mRNA level in A549 cells co-cultured with THP-1 macrophages was very low and independent of oxygen levels. In contrast, a larger amount of IL-1 $\beta$ mRNA was expressed in THP- 1 macrophages and the expression was hypoxia-dependent (Fig. 3B). IL-1 $\beta$ neutralizing antibody inhibited secretion of TGF- $\beta 1$ protein in co-culture media under hypoxia (Fig. 3C).

Thus, hypoxia stimulated THP-1 macrophages to up-regulate the IL- $1 \beta$. Then the IL-1 $\beta$ from THP- 1 macrophages acted on A549 cells and THP-1 macrophages to increase the production of TGF- $\beta 1$, and then the TGF- $\beta 1$ from both cells induced and promoted the EMT of A549 cells (Fig. 4).

In conclusion, our study indicated that the interaction between type II pneumocytes and macrophages under hypoxia was necessary for EMT of the type II pneumocytes, which potentially contribute to the development of pulmonary fibrosis.

\section{References}

[1] T.E. King Jr., A. Pardo, M. Selman, Idiopathic pulmonary fibrosis, Lancet 378 (2011) 1949-1961.

[2] G.A. Margaritopoulos, M. Romagnoli, V. Poletti, N.M. Siafakas, A.U. Wells, K.M. Antoniou, Recent advances in the pathogenesis and clinical evaluation of pulmonary fibrosis, Eur. Respir. Rev. 21 (2012) 48-56.

[3] B.C. Willis, Z. Borok, TGF- $\beta$-induced EMT: mechanisms and implications for fibrotic lung disease, Am. J. Physiol. Lung Cell. Mol. Physiol. 293 (2007) 525534.

[4] J. Zavadil, E.P. Zavadil, TGF-beta and epithelial-to-mesenchymal transitions, Oncogene 24 (2005) 5764-5774.

[5] C.J. Scotton, R.C. Chambers, Molecular targets in pulmonary fibrosis: the myofibroblast in focus, Chest 132 (2007) 1311-1321.

[6] B.C. Willis, J.M. Liebler, K. Luby-Phelps, A.G. Nicholson, E.D. Crandall, R.M. du Bois, Z. Borok, Induction of epithelial-mesenchymal transition in alveolar epithelial cells by transforming growth factor-beta1: potential role in idiopathic pulmonary fibrosis, Am. J. Pathol. 166 (2005) 1321-1332.

[7] P.J. Sime, Z. Xing, F.L. Graham, K.G. Csaky, J. Gauldie, Adenovector-mediated gene transfer of active transforming growth factor-beta 1 induces prolonged severe fibrosis in rat lung, J. Clin. Invest. 100 (1997) 768-776.

[8] V.L. Capelozzi, E.P. Faludi, A.B. Balthazar, M. Fernezlian Sde, J.V. Filho, E.R Parra, Bronchoalveolar lavage improves diagnostic accuracy in patients with diffuse lung disease, Diagn. Cytopathol. 41 (2013) 1-8.

[9] J. Domagała-Kulawik, T. Skirecki, M. Maskey-Warzechowska, H. GrubekJaworska, R. Chazan, Bronchoalveolar lavage total cell count in interstitial lung diseases - does it matter?, Inflammation 35 (2012) 803-809

[10] H.P. Hauber, P. Zabel, PAS staining of bronchoalveolar lavage cells for differential diagnosis of interstitial lung disease, Diagn. Pathol. 4 (2009) 13.

[11] M. Kawata, D. Koinuma, T. Ogami, K. Umezawa, C. Iwata, T. Watabe, K. Miyazono, TGF- $\beta$-induced epithelial-mesenchymal transition of A549 lung adenocarcinoma cells is enhanced by pro-inflammatory cytokines derived from RAW 264.7 macrophage cells, J. Biochem. 151 (2012) 205-216.

[12] A. Tzouvelekis, V. Harokopos, T. Paparountas, N. Oikonomou, A. Chatziioannou, G. Vilaras, E. Tsiambas, A. Karameris, D. Bouros, V. Aidinis, Comparative expression profiling in pulmonary fibrosis suggests a role of hypoxia-inducible factor-1alpha in disease pathogenesis, Am. J. Respir. Crit. Care Med. 176 (2007) 1108-1119.

[13] M.A. Rahat, H. Bitterman, N. Lahat, Molecular mechanisms regulating macrophage response to hypoxia, Front. Immunol. 2 (2011) 45.

[14] I.Y. Adamson, L. Young, D.H. Bowden, Relationship of alveolar epithelial injury and repair to the induction of pulmonary fibrosis, Am. J. Pathol. 130 (1988) 377-383.

[15] R. Kalluri, E.G. Neilson, Epithelial-mesenchymal transition and its implications for fibrosis, J. Clin. Invest. 112 (2003) 1776-1784.

[16] D.F. Higgins, K. Kimura, M. Iwano, V.H. Haase, Hypoxia-inducible factor signaling in the development of tissue fibrosis, Cell Cycle 7 (2008) 1128-1132.

[17] G. Zhou, L.A. Dada, J.I. Sznajder, Regulation of alveolar epithelial function by hypoxia, Eur. Respir. J. 31 (2008) 1107-1113.

[18] C. Madjdpour, U.R. Jewell, S. Kneller, U. Ziegler, R. Schwendener, C. Booy, C. Kläusli, T. Pasch, R.C. Schimmer, B. Beck-Schimmer, Decreased alveolar oxygen induces lung inflammation, Am. J. Physiol. Lung Cell. Mol. Physiol. 284 (2003) 360-367.

[19] L. Farkas, J. Gauldie, N.F. Voelkel, M. Kolb, Pulmonary hypertension and idiopathic pulmonary fibrosis: a tale of angiogenesis, apoptosis, and growth factors, Am. J. Respir. Cell Mol. Biol. 45 (2011) 1-15.

[20] S. Krick, B.G. Eul, J. Hänze, R. Savai, F. Grimminger, W. Seeger, F. Rose, Role of hypoxia-inducible factor-1alpha in hypoxia-induced apoptosis of primary alveolar epithelial type II cells, Am. J. Respir. Cell Mol. Biol. 32 (2005) 395-403.

[21] A.J. Majmundar, W.J. Wong, M.C. Simon, Hypoxia-inducible factors and the response to hypoxic stress, Mol. Cell 40 (2010) 294-309. 
[22] S.H. Phan, Biology of fibroblasts and myofibroblasts, Proc. Am. Thorac. Soc. 5 (2008) 334-337.

[23] Y.D. Xu, J. Hua, A. Mui, R. O'Connor, G. Grotendorst, N. Khalil, Release of biologically active TGF-beta 1 by alveolar epithelial cells results in pulmonary fibrosis, Am. J. Physiol. Lung Cell. Mol. Physiol. 285 (2003) 527-539.

[24] K.K. Kim, M.C. Kugler, P.J. Wolters, L. Robillard, M.G. Galvez, A.N. Brumwell, D. Sheppard, H.A. Chapman, Alveolar epithelial cell mesenchymal transition develops in vivo during pulmonary fibrosis and is regulated by the extracellular matrix, Proc. Natl. Acad. Sci. U.S.A. 103 (2006) 13180-13185.
[25] J. Theys, B. Jutten, R. Habets, K. Paesmans, A.J. Groot, P. Lambin, B.J. Wouters, G. Lammering, M. Vooijs, E-cadherin loss associated with EMT promotes radioresistance in human tumor cells, Radiother. Oncol. 99 (2011) 392-397.

[26] L.A. Borthwick, E.I. McIlroy, M.R. Gorowiec, M. Brodlie, G.E. Johnson, C. Ward, J.L. Lordan, P.A. Corris, J.A. Kirby, A.J. Fisher, Inflammation and epithelial to mesenchymal transition in lung transplant recipients: role in dysregulated epithelial wound repair, Am. J. Transplant. 10 (2010) 498-509. 